\title{
ON PASCAL OVALS
}

\author{
OLGA FERNANDES
}

(Received 29 November 1990)

Communicated by T. E. Hall

\begin{abstract}
In this paper we prove that if an oval in a finite projective plane of order $n \equiv 3(\bmod 4)$ has the four point Pascal property and if each of its tangents and secants has the five point Pascal property, then the plane is Pappian and the oval is a conic.

We also establish results concerning Ostrom conics and ovals with the three point and four point Pascal properties.

1991 Mathematics subject classification (Amer. Math. Soc.): primary 51 E 15; secondary 51 A 25.
\end{abstract}

\section{Introduction}

Buekenhout [3] has proved that if a projective plane contains a Pascal oval then the plane is Pappian and the oval is a conic. This has been re-proved by Artzy [1] (for a non-central oval), Rigby [10], and others. The conclusion of Buekenhout's result has been established with weakened hypotheses by Korchmáros [7] (for finite planes) and by Hofmann [6].

Hofmann's assumption is that the oval has the five point Pascal property. We weaken Hofmann's hypothesis for the case of an oval in a finite projective plane of order $n \equiv 3(\bmod 4)$ and prove that if an oval in such a plane has the four point Pascal property and if each of its tangents and secants has the five point Pascal property, then the plane is Pappian and the oval is a conic (Theorem 4.1). This is our main result.

We also establish, in Sections 5 and 6, results concerning Ostrom conics

(C) 1993 Australian Mathematical Society 0263-6115/93\$A2.00+0.00 
and ovals with the three point and four point Pascal properties.

We assume throughout this paper that the plane is finite and of odd order. In certain results we further assume that the plane is of order $n \equiv 3(\bmod 4)$.

\section{Background}

For relevant definitions and results concerning ovals see $[9,6,5]$.

If the three diagonal points [6, p. 146] of a non-degenerate hexagon inscribed in an oval are on a line $p$, then the hexagon is a Pascal hexagon and $p$ is its Pascal line [10, p. 1463]. A line $p$ is a Pascal line of an oval, if, whenever two diagonal points of a non-degenerate hexagon inscribed in the oval are on $p$ then the third diagonal point of the hexagon is also on $p$. A line $q$ has the five point (four point, three point) Pascal property if, whenever two diagonal points of a non-degenerate five point (four point, three point) hexagon inscribed in the oval are on $q$ then the third diagonal point of the hexagon is also on $q$. An oval has the five point (four point, three point) Pascal property if every line of the plane has the five point (four point, three point) Pascal property.

A line is non-exterior if it is a secant or tangent of the oval.

We note some useful results. Result 2.1 , on harmonic ovals, is established at the commencement of the proof of Theorem 4.2 in [5] and is valid for all odd orders of the plane.

2.1. An oval which has the four point Pascal property is harmonic (or has Property A1, or satisfies Assumption A1).

For the definition of a harmonic oval see [8, pp. 190, 191].

We now coordinatize the plane with reference to an oval $\mathscr{O}$ using Artzy's method (see [1]) and Hofmann's modification of Artzy's notation (see [6, p. 144]); except that we write the successive vertices of inscribed hexagons in order, as in $[10,5]$, and use angular brackets for points on the line $(\infty)(0)$. Thus, $\langle m\rangle$ is $(1)(m) \cap(\infty)(0)$.

The algebraic system $(S,+, \cdot)$, where $S \cup\{\infty\}$ is the coordinatizing set, is a double loop. If the oval has the four point Pascal property and if each of its non-exterior lines has the five point Pascal property then it is readily observed that the proofs of Propositions 5, 6, 8, 9 in [6] are valid. Accordingly:

2.2. The commutative loops $(S,+)$ and $(S-\{0\}, \cdot)$ have the alternative and inversive properties [6, Propositions $5,6,8]$. Also, they satisfy the Moufang identities. In particular:

$$
a+(b+(a+c))=((a+a)+b)+c=((a+b)+a)+c
$$

[6, Proposition 9]. 
(ii)

$$
(a+b)+(a+c)=a+(a+(b+c))=(a+a)+(b+c)
$$

This follows from (i) by [2, Lemma 3.1].

We re-prove (ii) in Lemma 3.2(ii).

With the same assumptions as for 2.2, the restriction of Proposition 7 of [6] to non-exterior lines is:

2.3. If $[m, k]$ is a non-exterior line then the point $(x, y)$ is on it if and only if $m x+y=k$.

\section{Preliminary lemmas}

Notations are as in Section 2. The letters $a, b, c, x, \ldots$ denote elements of $S$ or $S-\{0\}$ depending on the context. The product $a b$ is frequently denoted by $a \cdot b$ or $a(b)$. Proofs of trivial cases are omitted.

Lемма 3.1. If an oval has the four point Pascal property then

(i) $a .-b=b .-a$ and $-a .-b=a b$.

(ii) $a .-1=-a$.

(iii) $(-a)^{-1}=-a^{-1}$.

(iv) $a .-a=-a^{2}$.

(v) $a .-a^{-1}=-1$.

(vi) The point $(x, 0)$ is on the line $[m, k]$ if and only if $m=k x^{-1}$.

(vii) The points $\langle 1\rangle,(0, x),(x, 0)$ are collinear.

(viii) The points $(-1),(0, x),(-x, 0)$ are collinear.

Proof. (i) If $b \neq a$ then, since $(a)(-a),(b)(-b)$ are distinct secants through $(0,0)$ it follows, by 2.1 , that the points $(a)(-b) \cap(-a)(b)$ and $(a)(b) \cap(-a)(-b)$ are on $(\infty)(0)$. Thus $\langle a .-b\rangle=\langle b .-a\rangle$ and $\langle a b\rangle=$ $\langle-a$. $-b\rangle$. If $b=a$ then $-a .-a=a a$ by [6, Proposition 4].

(ii), (iii) are readily deduced from (i).

(iv) The polar of $\left\langle a^{2}\right\rangle$ is $(a)(-a)$ (see [6, Proposition 4]). The point $(1)\left(-a^{2}\right) \cap\left(a^{2}\right)(-1)$ is on the polars of both $(0,0)$ and $\left\langle a^{2}\right\rangle$. Thus $\left\langle-a^{2}\right\rangle=$ $\langle a .-a\rangle$.

(v) follows from (iii) and the fact that the point $(a)\left(-a^{-1}\right) \cap(-a)\left(a^{-1}\right)$ is on the polars of both $(0,0)$ and $\langle 1\rangle$.

(vi) follows from the collinearity of the diagonal points of the Pascal hexagon $(\infty)(\infty)(0)(0)(k)\left(x^{-1}\right)$.

(vii), (viii) are proved by using the Pascal hexagons $(\infty)(\infty)(0)(0)(x)\left(x^{-1}\right)$ and $(\infty)(\infty)(0)(0)(x)\left(-x^{-1}\right)$ respectively. 
In the Lemmas that follow, namely Lemmas 3.2, 3.3, 3.4, 3.5, 3.6, 3.7, we assume that the oval $\mathscr{O}$ has the four point Pascal property and that each of its non-exterior lines has the five point Pascal property.

LEMMA 3.2.

(i) $-(a+b)=-a-b$.

(ii) $(a+b)+(a+c)=(a+a)+(b+c) ;(a+b)+(a+b)=(a+a)+(b+b)$; $(a+b)+(a-b)=a+a$.

(iii) $a .-b=-a b$.

(iv) $(a b)^{-1}=a^{-1} b^{-1}$.

(v) $(x, 0)$ is on $[m, k]$ if and only if $m x=k$.

(vi) $\left(a^{-1}+b^{-1}, 0\right)$ is on $(a)(b)$ if and only if $(a+b, 0)$ is on $\left(a^{-1}\right)\left(b^{-1}\right)$.

Proof. (i) follows from the Pascal hexagon $(0)(0)(a)(-a-b)(a+b)(-b)$. (ii) $a+b=a+((b+c)-c)=a+((b+c)+(a+(-a-c)))=((a+a)+$ $(b+c))+(-a-c)$ by $2.2(\mathrm{i})$. The proof is easily completed.

(iii) We have $(-a b) a^{-1}=(a b) \cdot-a^{-1}=(-b \cdot-a) \cdot(-a)^{-1}=-b$. Hence $a .-b=-a b$.

The proofs of (iv), (v), (vi) are left to the reader.

In the proofs of the Lemmas that follow we frequently use Lemmas 3.1, 3.2 and the results of Section 2 without explicitly referring to them.

In Lemmas 3.3, 3.4, 3.5 we use the following notation.

Notation. $(\infty),(0)$ are denoted by $X, Y$ respectively. $A, B$ are (not necessarily distinct) points of $\mathscr{O}-\{X, Y\}, E$ is a point of $X Y$ such that $E \neq X, E \neq Y$ and $E \neq X Y \cap A B . A^{\prime}=A E \cap \mathscr{O}, B^{\prime}=B E \cap \mathscr{O}$, $P=A B \cap X X, Q=A B \cap Y Y, Q^{\prime}=A^{\prime} B^{\prime} \cap X X, P^{\prime}=A^{\prime} B^{\prime} \cap Y Y$.

LEMMA 3.3. Let the plane be of order $n \equiv 3(\bmod 4)$. Then $(0)(0) \cap$ $(a)(b)=\left(a^{-1}+b^{-1}, 0\right)$, equivalently $\left(a^{-1}+b^{-1}, 0\right)$ is on $(a)(b)$, for all $a, b \in S-\{0\}$.

PROOF. If $b=a^{-1}$ this follows from the collinearity of $\langle 1\rangle,\left(0, a+a^{-1}\right)$, $\left(a+a^{-1}, 0\right)$. If $b \neq a^{-1}$, let $\langle 1\rangle=E,(a)=A,(b)=B$. Then $P=$ $(0, a+b), A^{\prime}=\left(a^{-1}\right), B^{\prime}=\left(b^{-1}\right), Q^{\prime}=\left(0, a^{-1}+b^{-1}\right)$.

CASE (1). Suppose $E P$ or $E Q$ is a non-exterior line and $b \neq a^{-1}$. If $E Q$ is non-exterior then, because it is the Pascal line of $(0)(0)(\infty)(b)(a)\left(a^{-1}\right)$ and consequently of $(\infty)(\infty)(0)\left(a^{-1}\right)\left(b^{-1}\right)(b)$, it follows that $Q, E, Q^{\prime}$ are collinear. Thus $Q=(0)(0) \cap(a)(b)=\left(a^{-1}+b^{-1}, 0\right)$ by Lemma 3.1(vii). If 
$E P$ is non-exterior then the required result follows from the Pascal hexagons $(\infty)(\infty)(0)(b)(a)\left(a^{-1}\right),(0)(0)(\infty)\left(a^{-1}\right)\left(b^{-1}\right)(b)$ and Lemmas 3.1(vii), 3.2(vi)

CASE (2). Suppose both $E P$ and $E Q$ are exterior lines and $b \neq a^{-1}$. Since $E$ is an exterior point, the line through $E$ and $X X \cap Y Y$ is exterior [9, Lemma 2.1]. There are $\frac{1}{2}(n-1)$ exterior lines through $E$ and $\frac{1}{2}(n+3)$ non-exterior lines through $P$. Furthermore, $E P$ is an exterior line and $E Y, P Y$ are secants. It follows that there exist at least two points $Q_{i} \neq Y, i=1,2$ of $Y Y$ such that $E Q_{i}, P Q_{i}$ are non-exterior lines. Let $P Q_{1} \cap \mathscr{O}=\{(x),(y)\}$. By Case $(1), Q_{1}=(0)(0) \cap(x)(y)=$ $\left(x^{-1}+y^{-1}, 0\right)$. Thus, by Lemma 3.2(vi), $(0)(0) \cap\left(x^{-1}\right)\left(y^{-1}\right)=(x+y, 0)=$ $(a+b, 0)$. Let $m=x y$. Now $P Q_{1}=[m, a+b]$ is the Pascal line of the hexagons $(\infty)(\infty)(0)(b)(a)\left(m a^{-1}\right)$ and $(0)(0)(\infty)\left(m a^{-1}\right)\left(m b^{-1}\right)(b)$. Hence, by Case (1), the point $Q_{1}=(0)(0) \cap\left(m a^{-1}\right)\left(m b^{-1}\right)=\left(m^{-1} a+\right.$ $\left.m^{-1} b, 0\right)=\left(x^{-1}+y^{-1}, 0\right)$. Now consider the line $\left[m^{-1}, m^{-1} a+m^{-1} b\right]=$ $\left[x^{-1} y^{-1}, x^{-1}+y^{-1}\right]=\left(x^{-1}\right)\left(y^{-1}\right)$. It is the Pascal line of the hexagons $(\infty)(\infty)(0)\left(m^{-1} b\right)\left(m^{-1} a\right)\left(a^{-1}\right)$ and $(0)(0)(\infty)\left(a^{-1}\right)\left(b^{-1}\right)\left(m^{-1} b\right)$. Thus $(0)(0) \cap\left(a^{-1}\right)\left(b^{-1}\right)$ is on $\left(x^{-1}\right)\left(y^{-1}\right)$. Since $(a+b, 0)$ is on $\left(x^{-1}\right)\left(y^{-1}\right)$ it follows that $(0)(0) \cap\left(a^{-1}\right)\left(b^{-1}\right)=(a+b, 0)$ and hence, by Lemma 3.2(vi), that $(0)(0) \cap(a)(b)=\left(a^{-1}+b^{-1}, 0\right)$.

LEMMA 3.4. Let the plane be of order $n \equiv 3(\bmod 4)$. Then, for any choice of $A, B, E$, the points $P, E, P^{\prime}$ are collinear.

Proof. The point $\langle-1\rangle$ in which the polar $(1)(-1)$ of $\langle 1\rangle$ meets $(\infty)(0)$ is interior [9, Theorem 2.2]. Since the point (1) of $\mathscr{O}$ has been selected arbitrarily we can take $E=\langle 1\rangle$ if $E$ is an exterior point and $E=\langle-1\rangle$ if $E$ is an interior point. Let $A=(a), B=(b)$. Then, according as $E=\langle \pm 1\rangle$, we have $A^{\prime}=\left( \pm a^{-1}\right), B^{\prime}=\left( \pm b^{-1}\right)$; so that, by Lemmas 3.1(iii), 3.2(i) and 3.3, the point $P^{\prime}=( \pm(a+b), 0)$. The collinearity of $P, E, P^{\prime}$ is immediate.

Lemma 3.5. Let the plane be of order $n \equiv 3(\bmod 4)$. Then $(S,+, \cdot)$ is distributive.

Proof. We show that $c(a+b)=c a+c b$ for all $a, b, c, \in S-\{0\}$. If $c=$ $a^{-1} b^{-1}$ this is Lemma 3.3. If $c \neq a^{-1} b^{-1}$ let $E=\left\langle c^{-1}\right\rangle, A=(a), B=(b)$. Thus $P=(0, a+b), A^{\prime}=\left(c^{-1} a^{-1}\right), B^{\prime}=\left(c^{-1} b^{-1}\right), P^{\prime}=(c a+c b, 0)$. Since $P, E, P^{\prime}$ are collinear it follows that $c^{-1}(c a+c b)=a+b$; and hence that $c(a+b)=c a+c b$.

Notation. In Lemmas $3.6,3.7$ we denote $((a+b)+1)-(b+(a+1))$ by $f(a, b)$. 
LEMMA 3.6.

(i) If $f(a, b)=0$ then $f(b, a)=0$.

(ii) If $f\left(a, b_{1}\right)=0$ and $f\left(a, b_{2}\right)=0$ then $f\left(a, b_{1}+b_{2}\right)=0$.

Proof. (i) We use 2.2. Assume $f(a, b)=0$. Let $a+b=k$. Then $k+1=b+(a+1)$. Hence

$$
\begin{aligned}
(a+(k+1))+(b+1) & =(a+(b+(a+1)))+(b+1) \\
& =((a+(a+b))+1)+(b+1) \\
& =((a+k)+1)+((-a+k)+1) \\
& =((a+k)+(-a+k))+(1+1)=(k+k)+(1+1) \\
& =(k+1)+(k+1)=(a+(k+1))+(-a+(k+1)) .
\end{aligned}
$$

It follows that $b+1=-a+(k+1)$. Hence $a+(b+1)=(a+b)+1$. The proof of (ii) is left to the reader.

LEMMA 3.7. Let the plane be of order $n \equiv 3(\bmod 4)$. Then $(S,+)$ is associative.

Proof. It suffices to prove that $f(a, b)=0$ for all $a, b \in S$. That $f(a, 0)=0$ and $f(0, b)=0$ is obvious. Pick $a \in S-\{0\}$. Let $P=$ $\left(0, a^{-1}\right)$. Let $\left[m, a^{-1}\right]$ be a non-exterior line through $P$. Define $b^{-1}=$ $m-a^{-1}$ for $m \neq a^{-1}$. Since $m$ has $(n+1) / 2$ values it follows that there are at least $(n-1) / 2$ values of $b^{-1}$ corresponding to $a^{-1}$. Now $f(a,-1)=0$ and $f(a,-a)=0$. If $b \neq-1$ and $b \neq-a$ then $(\infty)(\infty)(0)\left(-b^{-1}\right)\left(a^{-1}+\right.$ $\left.b^{-1}\right)(1)$ and $(\infty)(\infty)\left(-b^{-1}\right)(0)\left(a^{-1}\right)(1)$ are Pascal hexagons with diagonal points on $\left[m, a^{-1}\right]$. Thus $(\infty)\left(-b^{-1}\right) \cap\left(a^{-1}\right)(1)=(-b, y)$, say, is on both $\left[a^{-1}, a^{-1}+1\right]$ and $\left[a^{-1}+b^{-1}, a^{-1}\right]$. Hence, by 2.3,y $=a^{-1} b+\left(a^{-1}+1\right)$ and $y=a^{-1}+b\left(a^{-1}+b^{-1}\right)$. Thus, by using Lemma 3.5, $b+(a+1)=1+(b+a)$; that is, $f(a, b)=0$. These values of $b$, together with $0,-1,-a$, constitute a set $\left\{b_{i}\right\}$, say, which contains at least $(n+1) / 2$ elements. It remains to prove that $f\left(a, b^{\prime}\right)=0$ for $b^{\prime} \notin\left\{b_{i}\right\}$. Let $\left(0, b^{\prime}\right)$ be on the tangent to at $\left(b^{\prime \prime}\right)$. If $b^{\prime \prime} \in\left\{b_{i}\right\}$, then, because $b^{\prime}=b^{\prime \prime}+b^{\prime \prime}$, we have $f\left(a, b^{\prime}\right)=0$ by Lemma 3.6(ii). Suppose $b^{\prime \prime} \notin\left\{b_{i}\right\}$. Since the number of points of the set $\mathscr{O}-\left\{\left(b_{i}\right)\right\}-\left\{(\infty),\left(b^{\prime \prime}\right)\right\}$ is at most $(n-3) / 2$ it follows that there is at least one secant through $\left(0, b^{\prime}\right)$ intersecting in points $\left(b_{1}\right),\left(b_{2}\right)$ of $\left\{\left(b_{i}\right)\right\}$. Thus, by Lemma 3.6(ii), $f\left(a, b^{\prime}\right)=f\left(a, b_{1}+b_{2}\right)=0$. 


\section{The main theorem}

THEOREM 4.1. Let the projective plane be of order $n \equiv 3(\bmod 4)$. If an oval has the four point Pascal property and if each of its tangents and secants has the five point Pascal property then the plane is Pappian and the oval is a conic.

Proof. It is immediate from 2.2 and Lemmas 3.7, 3.5 that $(S,+, \cdot)$ is a finite alternative division ring. It is thus a field. Since $(S,+)$ and $(S-\{0\}, \cdot)$ are Abelian groups it is readily deduced, (e.g. by reversing the argument in the latter part of the proof of Lemma 2 in [1]), that $(\infty)(\infty)$ and $(\infty)(0)$ are Pascal lines of the oval. Since $(\infty),(0)$ have been selected arbitrarily it follows that every tangent and every secant of the oval is a Pascal line. That the plane is Pappian and the oval a conic is immediate from [7].

\section{The Ostrom conic}

The definition of an Ostrom conic (see [9, p. 425] and [4, p. 272]) can be abbreviated thus:

THEOREM 5.1. An oval $\mathcal{O}$ in a finite projective plane of odd order is an Ostrom conic if each of its exterior and interior points is harmonic.

Proof. For the definition and some properties of harmonic points and lines see [4]. Define a $1-1$ correspondence $\alpha$ between the points and lines of the plane by associating each point of $\mathscr{\theta}$ with the tangent at the point, and each exterior and interior point with its harmonic line. Let points $P, Q$ be such that $Q$ is on $\alpha(P)$. That $P$ is on $\alpha(Q)$ is obvious if $P$ or $Q$ is on $\mathscr{O}$; and is immediate from [4, Theorem 3.1, (iv), (v)] if $P$ and $Q$ are not on $\mathscr{O}$ and at least one of them is exterior. If $P, Q$ are interior points then pick a point $A$ which is on $\mathscr{O}$ but not on $P Q$. Let $Q A \cap \mathscr{O}=B$, $P A \cap \mathscr{O}=A^{\prime}, P B \cap \mathscr{O}=B^{\prime}$. Since $P$ is harmonic, $A^{\prime} B^{\prime}$ goes through $Q$; and consequently $P=A A^{\prime} \cap B B^{\prime}$ is on $\alpha(Q)$. Thus $\alpha$ is a polarity of the plane. By [4, Corollary, p. 273] its only absolute points are the points of $\mathscr{O}$. Therefore $\theta$ is an Ostrom conic (see $[4$, p. 272]).

REMARK. Theorem 5.1 was motivated by Ostrom's Theorem 2.8 in [9].

THEOREM 5.2. An Ostrom conic in a finite projective plane of odd order has the first type of four point Pascal property. 
Proof. For the definition of an oval which has the first type of four point Pascal property see [5, p. 299]. Let $A A A^{\prime} B B B^{\prime}$ be a four point hexagon inscribed in the Ostrom conic. The points $A A^{\prime} \cap B B^{\prime}, A B^{\prime} \cap A^{\prime} B$ are on the harmonic line of $A B \cap A^{\prime} B^{\prime}$. Furthermore, by [4, Theorem 3.1(iv)], the point $A A \cap B B$ is also on this line. The required result is immediate.

THEOREM 5.3. If an oval in a projective plane of order $n \equiv 3(\bmod 4)$ has the four point Pascal property then it is an Ostrom conic.

PROoF. In the proof of Theorem 4.2 in [5] it is shown that the oval satisfies the hypothesis of $[9$, Lemma 2.2]. Therefore the poles of secants through an interior point are collinear. Hence, by considering the Pascal hexagons $A A A^{\prime} B B B^{\prime}, A^{\prime} A^{\prime} A B^{\prime} B^{\prime} B$, where $A B, A^{\prime} B^{\prime}$ are secants through an interior point, it is easy to prove that each interior point is harmonic. Furthermore, since the oval is harmonic (2.1), each exterior point is harmonic [4, p. 272]. It follows, by Theorem 5.1, that the oval is an Ostrom conic.

\section{Inscribed and circumscribed triangles}

Theorem 6.1. Let $A, B, C$ be distinct points of an oval in a projective plane of odd order $n$. Let $B B \cap C C=A^{\prime}, C C \cap A A=B^{\prime}, A A \cap B B=C^{\prime}$.

(i) If the oval has the three point Pascal property then the triangles $A B C$, $A^{\prime} B^{\prime} C^{\prime}$ are axially perspective.

(ii) If $n \equiv 3(\bmod 4)$ and the oval has the four point Pascal property then the triangles $A B C, A^{\prime} B^{\prime} C^{\prime}$ are centrally perspective.

Proof. The triangles are axially perspective if $A A \cap B C, B B \cap C A$, $C C \cap A B$ are collinear; and centrally perspective if $A A^{\prime}, B B^{\prime}, C C^{\prime}$ are concurrent. Hence (i) follows from the collinearity of the diagonal points of the Pascal hexagon $A A B B C C$. (ii) is proved by using (i) (because an oval which has the four point Pascal property also has the three point Pascal property); Theorem 5.3; and the fact that $A, B, C, A^{\prime}, B^{\prime}, C^{\prime}$ are the images of $A A, B B, C C, B C, C A, A B$ respectively under the polarity described in Theorem 5.1.

REMARK. Segre $[11$, p. 414$]$ has proved the concurrence of $A A^{\prime}, B B^{\prime}, C C^{\prime}$, for the case of an oval in a finite Desarguesian plane of odd order, as a preliminary to establishing his well-known theorem that such an oval is a conic. 


\section{References}

[1] R. Artzy, 'Pascal's theorem on an oval', Amer. Math. Monthly 75 (1968), 143-146.

[2] R. H. Bruck, A survey of binary systems (Springer, Berlin, 1958).

[3] F. Buekenhout, 'Plans projectifs à ovoïdes Pascaliens', Arch. Math. 17 (1966), 89-93.

[4] O. Fernandes, 'Harmonic points and the intersections of ovals', Geom. Dedicata 19 (1985), 271-276.

[5] - , 'On an oval with the four point Pascalian property', Canad. Math. Bull. 27 (1984), 295-300.

[6] C. E. Hofmann, 'Specializations of Pascal's theorem on an oval', J. Geom. 1 (1971), 143-153.

[7] G. Korchmáros, 'Una generalizzazione del teorema di F. Buekenhout sulle ovali pascaliane', Boll. Un. Mat. Ital. 18 (1981), 673-687.

[8] T. G. Ostrom, 'Conicoids: conic-like figures in non-Pappian planes', in: Geometry-von Staudt's point of view (eds. P. Plaumann and K. Strambach) (D. Reidel, Dordrecht, 1981) pp. 175-196.

[9] __, 'Ovals, dualities and Desargues's theorem', Canad. J. Math. 7 (1955), 417-431.

[10] J. F. Rigby, 'Pascal ovals in projective planes', Canad. J. Math. 21 (1969), 1462-1476.

[11] B. Segre, 'Ovals in a finite projective plane', Canad. J. Math. 7 (1955), 414-416.

\section{St. Patrick's Town}

Sholapur Road

Poona 411013

India 